\title{
Nematode assemblages from Mountain Pine (Pinus mugo Turra) communities in Pirin Mountain, Bulgaria
}

\author{
Y. MINCHEVA, S. LAZAROVA, V. PENEVA
} Department of Biodiversity, Central Laboratory of General Ecology, Bulgarian Academy of Sciences, 2 Gagarin
Street, 1113 Sofia, Bulgaria, E-mail: vpeneva@ecolab.bas.bg

\begin{abstract}
Summary
The structural and functional diversity of nematode communities from Pinus mugo sites in Pirin Mountain was assessed. The sampling plots represent different levels of disturbance - next to and outside ski runs. Nematode assemblages from this habitat can be characterised with high diversity (in total, 104 taxa and richness ranging from 41 to 65 genera per site). In general, the trophic structure was dominated by omnivorous and bacterial feeders coupled with low proportion of plant feeding nematodes. The high values of Maturity indices and the results from faunal analysis indicate relatively stable environment and undisturbed conditions for all sites. No clear correlation between the nematode assemblages' characteristics and the level of disturbance (position of the site in relation to ski runs) was revealed.
\end{abstract}

Keywords: diversity; ecological indices; life strategies; Pinus mugo; nematodes; trophic structure

\section{Introduction}

The human pressure on upland ecosystems has been increasing during the last decades (Kuzniar et al., 2004). It has a negative impact on ecosystem functioning and often leads to habitat fragmentation and loss of biodiversity. High mountain regions represent fragile and vulnerable ecosystems with a slow recovery after disturbance caused by both natural and human-driven factors. Mountain Pine (Pinus mugo Turra) shrublands are of great conservation value and have been included in the Habitat Directive (Directive 92/43/EEC). They protect soils against erosion and retard avalanching. However, outside the native ranges of distribution, $P$. mugo is regarded as an invasive species (Jørgensen, 2006).

Nematodes (free living and plant parasites) are suitable for environmental change assessment. The indicator potential of nematodes has been used previously to estimate soil characteristics and soil health (de Goede \& Bongers, 1994; Neher, 2001; Yeates, 2003), climate change (Ruess et al., 1999a, b; Sohlenius \& Boström, 1999a, b), heavy metal pollution (Korthals et al.,1996a, b), and habitat disturbance (Niblack, 1989). In alpine areas, nematode assemblages have been studied with regard to spatial variation between sites and influence of microclimate (Hoschitz, 2003; Hoschitz \& Kaufmann, 2004a, b).

Data on composition and structure of nematode communities from $P$. mugo habitats are insufficient and fragmentary (Tsaryk \& Kozlowsky, 1999; Lazarova, 2000; Ilieva, 2007,).

In the present study, we compared taxonomic composition and assemblage parameters (diversity and functional group distribution) of nematodes from seven $P$. mugo sites.

\section{Materials and methods}

\section{Site description}

The study area is situated in northern Pirin Mountains within the Pirin National Park, above the mountain resort of Bansko. Seven plots of mature, over hundred years old Mountain Pine shrub lands were sampled (Table 1). Yulen, Dolno Vassilashko Lake and Academica sites were not or slightly disturbed by anthropogenic influence, though Yulen's site was naturally fragmented. The rest of the sites Plato, Todorka 1, Todorka 2 and Todorka 3 - were situated next to the ski runs in the sub-alpine belt of Todorka peak. The soil type is Humic Cambisols on granite substratum. The average annual temperature is $3.3-3.7{ }^{\circ} \mathrm{C}$, the precipitation rate - $1200-1270 \mathrm{~mm}$ per year (Raev, 2005).

\section{Sampling, nematode extraction and identification} A total of 70 samples were collected in July 2005 from the $P$. mugo sites with 10 replicates per site. The samples were taken from $0-15-20 \mathrm{~cm}$ depth after removing the litter. Nematodes were recovered from $100 \mathrm{~cm}^{3}$ soil by sieving 
Table 1. Description of the studied Pinus mugo sites

\begin{tabular}{|c|c|c|c|c|c|c|}
\hline \multirow{2}{*}{ Locality } & \multirow{2}{*}{ Abbreviations } & \multicolumn{2}{|c|}{ Coordinates } & \multirow{2}{*}{$\begin{array}{l}\text { Elevation } \\
\text { meters } \\
\text { (a.s.l.) }\end{array}$} & \multirow[t]{2}{*}{ Exposition } & \multirow[t]{2}{*}{ Vegetation cover } \\
\hline & & $\mathrm{N}$ & $\mathrm{E}$ & & & \\
\hline Yulen & $\mathrm{uM}$ & $41^{\circ} 41^{\prime} 33.7^{\prime \prime}$ & $023^{\circ} 28^{\prime} 39.3^{\prime \prime}$ & 2160 & $\mathrm{~W}$ & $\begin{array}{l}\text { P. mugo, Festuca valida (Uechtr. Penzes), } \\
\text { Luzulla sp., Juniperus sibirica (Burgsd.), } \\
\text { Vaccinium myrtillus (L.) }\end{array}$ \\
\hline Dolno Vassilashko lake & dvM & $41^{\circ} 44^{\prime} 32^{\prime \prime}$ & $023^{\circ} 27^{\prime} 09^{\prime \prime}$ & 2135 & SE & P. mugo, Thymus sp., F. valida \\
\hline Plato & $\mathrm{pM}$ & $41^{\circ} 45^{\prime} 27.6^{\prime \prime}$ & $023^{\circ} 26^{\prime} 29.6^{\prime \prime}$ & 2375 & NE & P. mugo, V. myrtillus, Luzulla sp. \\
\hline Todorka & $\mathrm{t} 1 \mathrm{M}$ & $41^{\circ} 45^{\prime} 45^{\prime}$ & $023^{\circ} 26^{\prime} 20^{\prime \prime}$ & 2400 & $\mathrm{NE}$ & P. mugo \\
\hline Todorka & $\mathrm{t} 2 \mathrm{M}$ & $41^{\circ} 45^{\prime} 47.3^{\prime \prime}$ & $023^{\circ} 26^{\prime} 16.7^{\prime \prime}$ & 2400 & NE & P. mugo \\
\hline Todorka & $\mathrm{t} 3 \mathrm{M}$ & $41^{\circ} 45^{\prime} 53.6^{\prime \prime}$ & $023^{\circ} 26^{\prime} 26.1^{\prime \prime}$ & 2200 & NE & P. mugo, V. myrtillus \\
\hline Academica & $\mathrm{aM}$ & $41^{\circ} 47^{\prime} 16.4^{\prime \prime}$ & $023^{\circ} 25^{\prime} 21.1^{\prime \prime}$ & 2140 & NE & P. mugo, Luzula albida (Hoffm.), Poa sp. \\
\hline
\end{tabular}

and decanting technique, using two sieves with apertures of $1 \mathrm{~mm}$ and $64 \mu \mathrm{m}$, respectively. The final extraction was on small-sized sieves (aperture ca. $500 \mu \mathrm{m}$ ) in water filled Baerman funnels for $48 \mathrm{~h}$. The remnants of roots were placed on the small sieves in order to extract higher percentage of the nematode populations. Extracted nematodes were heat killed at $60{ }^{\circ} \mathrm{C}$, fixed in $4 \%$ formaldehyde and counted. At least 200 specimens per sample were processed in anhydrous glycerine and mounted on permanent slides (Seinhorst, 1959) for identification.

\section{Community parameters and analyses}

Based on their relative abundance, the nematode genera were divided into eudominants $(40-100 \%)$, dominants $(12.5-$ $39.9 \%)$, subdominants $(4-12.4 \%)$, recedents $(1.3-3.9 \%)$ subrecedents $(0.1-1.2 \%)$ according to Engelmann (1978).

The nematode taxa were assigned to five feeding groups following the classification of Yeates et al. (1993). Plant feeding nematodes belonging to the sub-group " $\mathrm{f}$ " were regarded separately from the groups "a-e"and denoted below as algal feeders (AF). The Maturity indices, MI and PPI (Bongers, 1990), MI $2-5$ (based on the cp groups 2-5, e.g. Korthals et al., 1996) and इMI (Yeates, 1994) were calculated. The following diversity measures: generic richness (S), Shannon's diversity index (H') and Simpson's concentration of dominance index (C)(Krebs, 1989) were calculated for each sample. Additionally, Enrichment index (EI), Channel index (CI) and Structure index (SI), (Ferris et al., 2001) were used to characterise the soil food webs.

The diversity parameters, similarity measures and cluster analysis were performed using the program BIODIV 5.2 (Baev \& Penev, 1995).

The linear-response model of Principal Components Analysis (PCA) based on generic abundance data, logtransformed prior to analyses was most appropriate for analysing the nematode communities of studied sites (gradient length in CA/DCA did not exceed 3.0 (4.0) SD, a general limits for the acceptance of linear models (ter Braak, 1996). Further, an indirect gradient analysis was applied in order to visualize the relation of generic composition and community measures. All gradient analyses and ordination graphs were performed using CANOCO version 4.5 (ter Braak \& Smilauer, 2002). Multiple comparisons of samples datasets were made with nonparametric Kruskal-Wallis $H$ test; significant differences were tested using the Mann-Whitney $U$ test (Statistica 8.0).

\section{Results and discussion}

Diversity, abundance, occurrence and dominance

A total of 104 taxa (a hundred determined to generic, three to subfamily and one to family level) were recorded from all studied sites, with the greatest number identified at Academica (aM, 65) and the least at Todorka ( $\mathrm{t} 3 \mathrm{M}$, 41)(Table 2). Thus, the nematode assemblages from the northern Pirin were characterised by high generic richness compared to the data from other Mountain Pine sites: 34 genera reported from Rila Mt. (Ilieva, 2007) and 46 - from Vitosha Mt. (Lazarova, 2000).

The total number of nematodes varied significantly among sites (Table 3) with average values ranging from 177 (at $\mathrm{uM}$ ) to 2257 (at t1M). With regard to their frequency of occurrence, the nematode taxa could be divided into the following groups: common - found at all seven sites (17); less common - found in four to six sites (34) or three sites (16); and rare - found in one or two sites (35) (Table 2). In general, Tylencholaimus (11.4\%), Aporcelaimellus (10.3 \%), Plectus (10.2\%), Metaporcelaimus (6.5\%), Eudorylaimus $(6.4 \%)$, Acrobeloides $(5.7 \%)$ were the most abundant in all sites. The taxonomic composition appeared rather uniform among sites, because half of the genera were common for the majority of sites $(4-7)$. Among them, only six genera were dominant for at least one plot (Acrobeloides, pM; Plectus, uM \& t2M; Tylencholaimus, t1M; Aporcelaimellus, dvM, pM \& t2M; Metaporcelaimus, t3M; Coomansus, t3M). Dominant structure of the Mountain Pine sites was composed by $1-3$ dominant and $4-8$ subdominant genera, with no eudominant genera present (see Table 2 for details). With few exceptions (Tylocephalus, Labronemella, Longidorella, Malenchus and Clarkus), all rare taxa which occurred in one to three sites, were present in very low numbers (subrecedents).

A similar pattern in dominance structure was observed for the nematode assemblages from Vitosha and Rila Mts (3 - 2 dominant and $4-3$ subdominant genera, respectively (Lazarova, 2000; Ilieva, 2007). Only three dominant genera (Plectus, Aporcelaimellus and Helicotylenchus) were common for the P. mugo sites in all three mountains. Characteristic features of the nematode assemblages from the other two studied regions were the high proportions of Alaimus and Tripyla in Vitosha and Malenchus in Rila Mts. The variation among the nematode communities in all studied plots was calculated with Principal Components 
Table 2. Mean relative abundance (A\% per $100 \mathrm{~cm}^{3}$ fresh substrate) and frequency ( $\mathrm{F} \%$ occurrence in the replicates) of nematode taxa from $P$. mugo sites

\begin{tabular}{|c|c|c|c|c|c|c|c|c|c|c|c|c|c|c|}
\hline \multirow[t]{2}{*}{ Genera } & \multicolumn{2}{|c|}{ Yulen } & \multicolumn{2}{|c|}{$\begin{array}{c}\text { Dolno Vassilashko } \\
\text { lake }\end{array}$} & \multicolumn{2}{|c|}{ Plato } & \multicolumn{2}{|c|}{ Todorka 1} & \multicolumn{2}{|c|}{ Todorka 2} & \multicolumn{2}{|c|}{ Todorka 3} & \multicolumn{2}{|c|}{ Academica } \\
\hline & A & $\mathrm{F}$ & A & $\mathrm{F}$ & $\mathrm{A}$ & $\mathrm{F}$ & $\mathrm{A}$ & $\mathrm{F}$ & $\mathrm{A}$ & $\mathrm{F}$ & $\mathrm{A}$ & $\mathrm{F}$ & $\mathrm{A}$ & $\mathrm{F}$ \\
\hline \multicolumn{15}{|l|}{ Bacterial Feeders } \\
\hline Acrobeloides & 3.0 & 90 & 8.3 & 100 & 14.4 & 90 & 0.5 & 40 & 8.2 & 90 & 2.0 & 70 & 3.4 & 100 \\
\hline Alaimus & 3.3 & 60 & 4.5 & 100 & 0.6 & 60 & 0.7 & 60 & 0.2 & 40 & 0.1 & 10 & 1.9 & 70 \\
\hline Amphidelus & 0.2 & 20 & - & - & - & - & - & - & - & - & - & - & - & - \\
\hline Anaplectus & 2.1 & 40 & 0.8 & 50 & 0.7 & 50 & 0.1 & 20 & - & - & - & - & 0.9 & 60 \\
\hline Aulolaimus & - & - & - & - & - & - & - & - & - & - & - & - & 0.1 & 30 \\
\hline Bastiania & 0.2 & 20 & 0.9 & 60 & 0.3 & 30 & 0.8 & 70 & + & 10 & + & 10 & 0.7 & 50 \\
\hline Bunonema & - & - & - & - & - & - & - & - & - & - & 0.1 & 10 & - & - \\
\hline Cephalobus & - & - & - & - & - & - & + & 10 & - & - & - & - & 1.2 & 80 \\
\hline Ceratoplectus & 0.2 & 20 & + & 10 & - & - & - & - & - & - & - & - & - & - \\
\hline Cervidellus & 0.7 & 40 & 2.7 & 70 & 0.6 & 60 & + & 10 & 0.1 & 10 & 0.1 & 10 & 2.1 & 90 \\
\hline Chiloplacus & 0.1 & 10 & 0.5 & 50 & - & - & 0.2 & 30 & - & - & - & - & 0.8 & 60 \\
\hline Chromadorina & - & - & 0.1 & 10 & - & - & - & - & - & - & - & - & - & - \\
\hline Cylindrolaimus & - & - & 0.3 & 30 & - & - & - & - & - & - & - & - & 0.2 & 20 \\
\hline Eucephalobus & 0.4 & 20 & 0.5 & 40 & - & - & - & - & - & - & 0.1 & 10 & 4.2 & 70 \\
\hline Eumonhystera & - & - & 0.2 & 50 & 0.2 & 40 & - & - & - & - & 0.1 & 20 & 0.4 & 50 \\
\hline Euteratocephalus & - & - & - & - & - & - & + & 10 & 0.2 & 10 & - & - & - & - \\
\hline Geomonhystera & - & - & 0.3 & 10 & 0.1 & 10 & - & - & - & - & - & - & 0.1 & 10 \\
\hline Heterocephalobus & - & - & 1 & 80 & 1.2 & 70 & 0.4 & 10 & - & - & - & - & 0.2 & 40 \\
\hline Metateratocephalus & 0.1 & 10 & 0.1 & 20 & 0.1 & 10 & - & - & 0.2 & 40 & 0.1 & 20 & 0.3 & 20 \\
\hline Monhystrella & - & - & - & - & - & - & - & - & - & - & 0.1 & 10 & - & - \\
\hline Odontolaimus & - & - & - & - & - & - & + & 10 & - & - & - & - & - & - \\
\hline Panagrolaimus & - & - & 0.1 & 20 & - & - & 0.1 & 10 & - & - & 0.6 & 20 & + & 10 \\
\hline Paramphidelus & 0.1 & 10 & 0.1 & 20 & - & - & - & - & - & - & - & - & 1.3 & 50 \\
\hline Pelodera & 0.5 & 10 & - & - & - & - & - & - & - & - & - & - & + & 10 \\
\hline Plectus & 15.6 & 100 & 8.9 & 100 & 7.4 & 100 & 4.7 & 100 & 15.4 & 100 & 13.1 & 100 & 6.3 & 100 \\
\hline Prismatolaimus & 0.1 & 20 & 0.9 & 60 & 1.1 & 70 & 2.2 & 100 & 0.7 & 40 & - & - & 1.0 & 70 \\
\hline Prodesmodora & 0.1 & 10 & + & 10 & 0.1 & 10 & 0.4 & 40 & + & 10 & - & - & 0.2 & 30 \\
\hline Rhabditis & - & - & 0.1 & 10 & - & - & - & - & - & - & 0.4 & 20 & - & - \\
\hline Rhabdolaimus & - & - & 0.1 & 20 & 1.6 & 40 & 9.2 & 100 & 0.7 & 60 & - & - & 0.6 & 60 \\
\hline Seleborca & - & - & + & 10 & - & - & 1.7 & 70 & - & - & - & - & 0.1 & 20 \\
\hline Stenonchulus & - & - & - & - & - & - & + & 10 & - & - & - & - & 0.1 & 20 \\
\hline Teratocephalus & 1.0 & 70 & 3.4 & 70 & 5.6 & 100 & 2.4 & 100 & 1.6 & 100 & 1.7 & 80 & 1.6 & 60 \\
\hline Theristus & - & - & - & - & - & - & - & - & - & - & - & - & + & 10 \\
\hline Tylocephalus & - & - & - & - & - & - & - & - & 0.1 & 10 & - & - & 1.3 & 60 \\
\hline Wilsonema & 0.1 & 10 & 0.3 & 30 & 0.1 & 20 & - & - & 0.1 & 10 & - & - & - & - \\
\hline \multicolumn{15}{|l|}{ Animal Parasites } \\
\hline Howardula & - & - & - & - & - & - & - & - & 0.3 & 10 & - & - & - & - \\
\hline Steinernema & 0.2 & 10 & 0.4 & 30 & 2.7 & 30 & - & - & 0.5 & 60 & 0.4 & 40 & 0.4 & 30 \\
\hline Mermithidae & 0.1 & 10 & - & - & - & - & - & - & - & - & - & - & + & 10 \\
\hline \multicolumn{15}{|l|}{ Fungal Feeders } \\
\hline Aphelenchoides & 1.1 & 50 & 5.3 & 100 & 5.4 & 100 & 3.2 & 100 & 2.5 & 90 & 4.7 & 100 & 4.2 & 100 \\
\hline Aphelenchus & - & - & - & - & 0.4 & 10 & 0.05 & 10 & - & - & 0.1 & 10 & - & - \\
\hline Deladenus & - & - & - & - & - & - & - & - & 0.3 & 20 & 0.7 & 40 & + & 10 \\
\hline Diphterophora & 0.6 & 30 & 0.8 & 70 & 0.3 & 60 & 0.6 & 50 & 1.8 & 70 & 0.3 & 40 & 0.1 & 40 \\
\hline Ditylenchus & 0.8 & 80 & 0.4 & 60 & 1.3 & 80 & 0.6 & 70 & 0.6 & 60 & 1.0 & 60 & 0.2 & 20 \\
\hline Dorylaimellus & 0.7 & 10 & 2.5 & 80 & 4.7 & 80 & 12.3 & 100 & 0.5 & 30 & - & - & - & - \\
\hline Hexatylus & - & - & 0.6 & 10 & - & - & 0.1 & 10 & - & - & 0.1 & 10 & 0.1 & 20 \\
\hline
\end{tabular}




\begin{tabular}{|c|c|c|c|c|c|c|c|c|c|c|c|c|c|c|}
\hline Nothotylenchus & - & - & 0.8 & 50 & + & 10 & - & - & - & - & 0.1 & 10 & - & \\
\hline Paraphelenchus & - & - & - & - & + & 10 & 0.1 & 10 & + & 10 & 0.0 & 0 & - & - \\
\hline Pseudhalenchus & 0.1 & 10 & 0.4 & 40 & 0.6 & 20 & 0.6 & 50 & 0.3 & 40 & 0.1 & 10 & - & - \\
\hline Safianema & - & - & + & 10 & 1.4 & 100 & 0.3 & 50 & 1.3 & 70 & - & - & - & 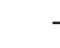 \\
\hline Tylencholaimellus & - & - & - & - & - & - & 0.05 & 10 & - & - & - & - & - & - \\
\hline Tylencholaimus & 2.3 & 50 & 7.1 & 100 & 5.7 & 80 & 18.9 & 100 & 10 & 100 & 2.8 & 60 & 33.3 & 100 \\
\hline Tylolaimophorus & 0.1 & 10 & 0.8 & 40 & - & - & 0.2 & 20 & - & - & - & - & - & - \\
\hline \multicolumn{15}{|l|}{ Omnivorous } \\
\hline Allodorylaimus & 0.2 & 30 & 0.3 & 10 & 0.7 & 60 & 0.5 & 40 & 0.8 & 30 & 0.5 & 40 & + & 10 \\
\hline Aporcelaimellus & 5.8 & 90 & 12.5 & 100 & 16.7 & 100 & 11.8 & 100 & 15.3 & 100 & 1.7 & 80 & 8.1 & 100 \\
\hline Aporcelaimus & 0.7 & 30 & 1.3 & 100 & 0.4 & 70 & 0.3 & 50 & 2.7 & 80 & 2.8 & 90 & 0.5 & 50 \\
\hline Dorylaimoides & 0.2 & 20 & 1.4 & 70 & 1.5 & 90 & 2.9 & 90 & 0.4 & 60 & 0.2 & 10 & 1.0 & 50 \\
\hline Enchodelus & 0.9 & 40 & 0.1 & 20 & 0.1 & 10 & - & - & 0.1 & 20 & 0.2 & 30 & 0.2 & 40 \\
\hline Epacrolaimus & 0.2 & 10 & - & - & - & - & - & - & - & - & - & - & - & - \\
\hline Epidorylaimus & - & - & - & - & + & 10 & 1.1 & 30 & 0.4 & 40 & - & - & 0.4 & 40 \\
\hline Eudorylaimus & 12.2 & 100 & 4.6 & 90 & 2.1 & 90 & 3.2 & 90 & 9.4 & 100 & 5.6 & 100 & 7.8 & 80 \\
\hline Labronemella & - & - & - & - & 1.4 & 60 & 0.6 & 70 & 0.5 & 20 & - & - & - & - \\
\hline Metaporcelaimus & 7.3 & 100 & 7.6 & 100 & 6.2 & 100 & + & 10 & 8.9 & 90 & 15.6 & 100 & + & 10 \\
\hline Microdorylaimus & - & - & - & - & - & - & - & - & - & - & - & - & 0.7 & 60 \\
\hline Morasia & - & - & - & - & - & - & + & 10 & - & - & - & - & - & - \\
\hline Oonaguntus & - & - & 0.9 & 60 & 0.3 & 50 & - & - & - & - & - & - & + & 10 \\
\hline Thonus & 5.9 & 80 & 7.5 & 80 & 1.1 & 50 & - & - & 5.1 & 50 & 11.5 & 100 & 2.3 & 80 \\
\hline \multicolumn{15}{|l|}{ Plant Feeders } \\
\hline Rotylenchus & 0.1 & 10 & 0.6 & 20 & 0.4 & 30 & - & - & - & - & - & - & - & - \\
\hline Pratylenchoides & - & - & - & - & 0.1 & 10 & - & - & + & 10 & - & - & - & - \\
\hline Pratylenchus & - & - & - & - & 0.1 & 10 & 0.8 & 70 & 0.5 & 40 & - & - & 0.2 & 10 \\
\hline Helicotylenchus & 4.3 & 40 & 0.5 & 50 & 2.2 & 60 & 1.7 & 40 & 0.1 & 10 & 1.0 & 50 & + & 10 \\
\hline Amplimerlinius & - & - & - & - & - & - & - & - & - & - & - & - & 0.1 & 10 \\
\hline Bitylenchus & - & - & - & - & 0.1 & 10 & 0.1 & 10 & - & - & - & - & - & - \\
\hline Cephalenchus & - & - & 0.2 & 30 & 0.1 & 20 & 0.1 & 10 & 0.4 & 30 & - & - & - & - \\
\hline Criconemoides & - & - & - & - & - & - & - & - & - & - & 0.1 & 10 & + & 10 \\
\hline Longidorella & 2.4 & 30 & 0.2 & 10 & - & - & - & - & - & - & - & - & 1.8 & 70 \\
\hline Longidorus & - & - & - & - & + & 10 & - & - & - & - & - & - & - & - \\
\hline Merlinius & 0.8 & 30 & 0.1 & 20 & 1.3 & 30 & + & 10 & 0.1 & 20 & - & - & - & - \\
\hline Mesocriconema & 0.1 & 10 & 0.1 & 30 & 0.2 & 30 & 2.9 & 80 & - & - & - & - & - & - \\
\hline Nagelus & - & - & 0.2 & 10 & - & - & - & - & - & - & - & - & + & 10 \\
\hline Paratylenchus & 0.8 & 50 & 1.6 & 80 & 1.5 & 60 & 1.3 & 80 & 1.2 & 90 & 1.7 & 70 & - & - \\
\hline Pungentus & - & - & 0.2 & 20 & - & - & - & - & - & - & - & - & + & 10 \\
\hline Trichodorus & - & - & - & - & - & - & - & - & - & - & - & - & 0.6 & 20 \\
\hline Tylenchorhinchus & + & 10 & + & 10 & 0.3 & 30 & 2.5 & 80 & - & - & - & - & 0.1 & 10 \\
\hline Basiria & 0.1 & 10 & 0.5 & 70 & 0.5 & 40 & 0.1 & 10 & - & - & - & - & 0.2 & 40 \\
\hline Coslenchus & 0.4 & 40 & - & - & 1.1 & 50 & - & - & 0.4 & 10 & 0.1 & 10 & + & 10 \\
\hline Filenchus & - & - & 1 & 80 & 0.2 & 20 & 0.5 & 70 & + & 10 & 0.3 & 40 & 0.3 & 30 \\
\hline Malenchus & - & - & + & 10 & - & - & - & - & - & - & 1.7 & 50 & 3.6 & 60 \\
\hline Miculenchus & - & - & - & - & 0.7 & 10 & 0.3 & 30 & 0.1 & 20 & - & - & 0.1 & 20 \\
\hline Ottolenchus & - & - & 0.5 & 30 & 0.6 & 40 & 0.1 & 20 & 0.2 & 40 & - & - & - & - \\
\hline Tylenchus & 4.4 & 80 & 1.3 & 70 & 3.1 & 100 & 2.4 & 90 & 3.7 & 100 & 4.5 & 100 & 1.4 & 90 \\
\hline Criconematinae & - & - & - & - & - & - & - & - & + & 10 & - & - & - & - \\
\hline Ecphyadophorinae & - & - & - & - & + & 10 & - & - & - & - & - & - & - & - \\
\hline Telotylenchidae & 0.1 & 10 & - & - & - & - & - & - & - & - & - & - & - & - \\
\hline \multicolumn{15}{|l|}{ Predators } \\
\hline Nygolaimus & 0.1 & 10 & - & - & - & - & + & 10 & - & - & - & - & - & - \\
\hline Paravulvus & 0.4 & 20 & - & - & 0.2 & 20 & 5.9 & 90 & - & - & - & - & 0.7 & 40 \\
\hline Clarkus & 3.7 & 70 & 2 & 50 & - & - & - & - & - & - & - & - & 0.1 & 20 \\
\hline
\end{tabular}




\begin{tabular}{lrrrrrrrrrrrrrr} 
Coomansus & 9.9 & 80 & - & - & - & - & + & 10 & 3.4 & 90 & 18.7 & 100 & 0.4 & 10 \\
Mylonchulus & - & - & - & - & + & 10 & - & - & - & - & - & - & 0.8 & 70 \\
Prionchulus & - & - & - & - & - & - & - & - & - & - & - & - & 0.2 & 30 \\
Solididens & 0.1 & 10 & + & 10 & - & - & 0.2 & 30 & - & - & - & - & - & - \\
Tripyla & 5 & 60 & - & - & 0.3 & 20 & - & - & + & 10 & 3.3 & 70 & - & - \\
Actinolaimoidinae & 0.1 & 10 & - & - & - & - & - & - & - & - & - & - & - & - \\
& & & & & & & & & & & & & \\
Dauer larvae & 0.5 & 50 & 1.4 & 70 & 0.1 & 10 & - & - & 0.4 & 30 & 1.9 & 60 & 0.7 & 90 \\
unidentified & - & - & + & 10 & 0.1 & 20 & - & - & - & - & + & 10 & + & 10 \\
\hline
\end{tabular}

Note: Values less than 0.05 marked with '+'.
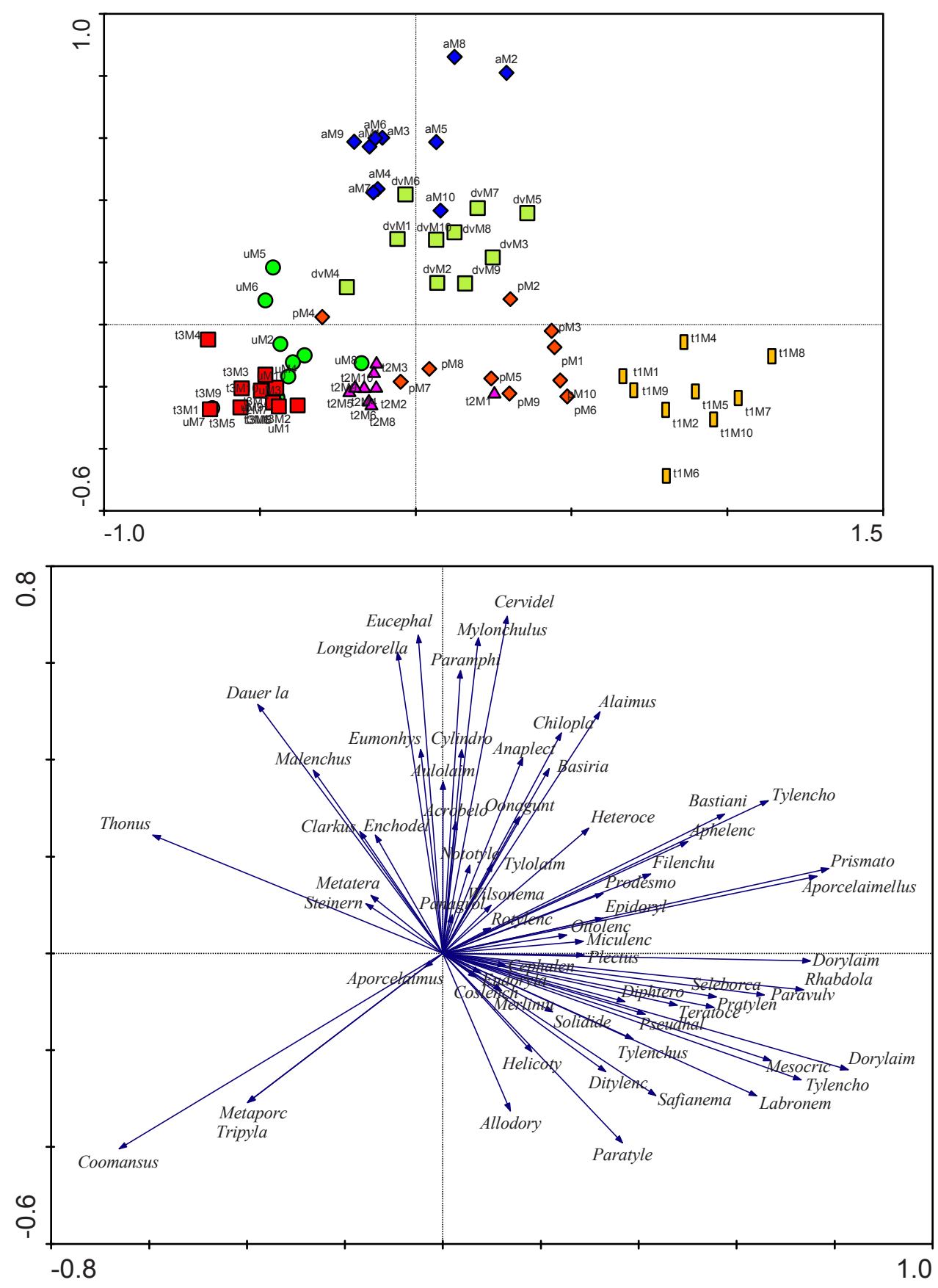

Fig. 1. Principal Component Analysis of the nematode communities based on generic abundance data: A - sites (abbreviations are the same as in Table 1); B - generic distribution 
Analysis, presented separately at Fig. 1a and Fig 1b, for sites and genera, respectively. The three ordination axes explain $39 \%$ of the variance of generic composition data. The first axis of the PCA (eigenvalue 0.210 ) represents a gradient from sites with the lowest abundance and diversity ( $\mathrm{uM}, \mathrm{t} 3 \mathrm{M}, \mathrm{t} 2 \mathrm{M}$ ) to sites with more diverse and numerous nematode communities (pM, dvM, aM and t1M) (see also Table 3). Further, the second axis of the PCA (0.091) divided the the sites of higher human impact - in close vicinity to ski runs ( $\mathrm{t} 3 \mathrm{M}, \mathrm{uM}, \mathrm{t} 2 \mathrm{M}, \mathrm{pM}$ and $\mathrm{t} 1 \mathrm{M})$, from those that were non disturbed (aM and dvM). The nematode communities of the latter two sites also formed well distinguisheable groups at the third ordination axis $(0.084$, figure not included), which revealed specific genera occurring at one of the two sites (Table 2). Among the common genera, Alaimus, Aporcelaimus, Diphtherophora, Filenchus, Heterocephalobus and Metaporcelaimus, prevailed significantly at dvM site $(\mathrm{p}<0.05)$ and Eucephalobus, Malenchus and Tylencholaimus - were significantly more abundant at aM site $(\mathrm{p}<0.05)$.

Classification procedure performed with the Sørensen percentage similarity index $\left({ }^{*}{ }^{*} I_{c s} * b\right)$ showed similar patterns (Fig 2). Two main groups of sites (clusters 1 and 2) represented a pattern close to the arrangement of the sites

Table 3. Community parametres of nematode assemblages from seven $P$. mugo sites: mean relative abundance (numbers per $100 \mathrm{~cm}^{3}$ fresh substrate), generic richness, the Shannon-Wiener diversity index (H') and Simpson's dominance index (C), trophic groups, colonizer-persistor (cp and pp) groups, the maturity indices (MI, $\Sigma$ MI, MI 2-5 and PPI), Channel (CI), Enrichment (EI) and Structure index (SI).

\begin{tabular}{|c|c|c|c|c|c|c|c|c|c|c|c|c|c|c|c|c|c|c|c|c|c|}
\hline & \multicolumn{3}{|c|}{ Yulen } & \multicolumn{3}{|c|}{ Dolno Vassilashko lake } & \multicolumn{3}{|c|}{ Plato } & \multicolumn{3}{|c|}{ Todorka 1} & \multicolumn{3}{|c|}{ Todorka 2} & \multicolumn{3}{|c|}{ Todorka 3} & \multicolumn{3}{|c|}{ Academica } \\
\hline & Mean & SD & & Mean & SD & & Mean & SD & & Mean & SD & & Mean & SD & & Mean & SD & & Mean & SD & \\
\hline Mean abundance & 177 & 69.6 & $\mathrm{a}$ & 727 & 244 & $\mathrm{~b}$ & 988 & 828 & $\mathrm{~b}$ & 2257 & 1589 & $\mathrm{c}$ & 357 & 227 & $\mathrm{~d}$ & 380 & 434 & ad & 717 & 484 & $\mathrm{~b}$ \\
\hline Generic richness & 20 & 6.6 & $\mathrm{a}$ & 30 & 5.38 & $\mathrm{~b}$ & 27 & 5.05 & $\mathrm{~b}$ & 26 & 2.85 & $\mathrm{~b}$ & 22 & 3.8 & $\mathrm{a}$ & 19 & 3.67 & $\mathrm{a}$ & 28 & 6.78 & $\mathrm{~b}$ \\
\hline $\mathrm{H}^{\prime}$ & 2.30 & 0.38 & ac & 2.66 & 0.32 & $\mathrm{~b}$ & 2.50 & 0.30 & $\mathrm{abc}$ & 2.52 & 0.16 & $\mathrm{ab}$ & 2.27 & 0.41 & $\mathrm{bc}$ & 2.28 & 0.25 & $\mathrm{c}$ & 2.36 & 0.28 & $a b c$ \\
\hline $\mathrm{C}$ & 0.15 & 0.05 & $a b c$ & 0.11 & 0.05 & $\mathrm{a}$ & 0.14 & 0.06 & $a b c$ & 0.12 & 0.03 & $a b c$ & 0.17 & 0.12 & $a b c$ & 0.15 & 0.05 & $\mathrm{bc}$ & 0.17 & 0.07 & $\mathrm{c}$ \\
\hline \multicolumn{22}{|l|}{ Trophic groups } \\
\hline Sum PF & 9.2 & 11 & $\mathrm{a}$ & 5.7 & 4.1 & $\mathrm{a}$ & 9.3 & 7.6 & $\mathrm{a}$ & 10 & 11 & $\mathrm{a}$ & 3.1 & 2.2 & $\mathrm{~b}$ & 4.8 & 3.5 & $\mathrm{ab}$ & 7.1 & 3.8 & $\mathrm{a}$ \\
\hline $\mathrm{PFb}$ & 0 & 0 & $\mathrm{a}$ & 0 & 0 & $\mathrm{a}$ & 0.2 & 0.5 & $a b$ & 0.8 & 1 & $\mathrm{~b}$ & 0.5 & 0.9 & $\mathrm{bc}$ & 0 & 0 & $\mathrm{a}$ & 0.2 & 0.5 & $\mathrm{ac}$ \\
\hline $\mathrm{PFc}$ & 4.5 & 9.2 & $\mathrm{a}$ & 1.1 & 2.1 & $\mathrm{a}$ & 2.5 & 4.5 & $\mathrm{a}$ & 1.7 & 4.2 & $\mathrm{ab}$ & 0.1 & 0.2 & $\mathrm{~b}$ & 1 & 1.7 & $\mathrm{ac}$ & 0.1 & 0.4 & $\mathrm{bc}$ \\
\hline PFd & 4.3 & 4.6 & $\mathrm{a}$ & 3.1 & 3.5 & $\mathrm{a}$ & 4 & 3.6 & $\mathrm{ab}$ & 7 & 7.4 & $\mathrm{~b}$ & 1.7 & 1 & $\mathrm{a}$ & 1.8 & 2.3 & $\mathrm{a}$ & 2.8 & 3 & $a b$ \\
\hline Pfe & 0.5 & 0.7 & $\mathrm{a}$ & 1.6 & 1.1 & $\mathrm{~b}$ & 2.5 & 3.3 & $\mathrm{ab}$ & 0.9 & 1 & $\mathrm{ab}$ & 0.7 & 1.4 & $\mathrm{a}$ & 2.1 & 2.8 & $a b$ & 4 & 4.7 & $\mathrm{~b}$ \\
\hline $\operatorname{PFf}(=\mathrm{AF})$ & 4.4 & 9.4 & $\mathrm{a}$ & 1.3 & 1 & $\mathrm{a}$ & 3.1 & 2.7 & $a b$ & 2.4 & 2 & $\mathrm{ab}$ & 3.7 & 3.7 & $\mathrm{ab}$ & 4.5 & 3.5 & $\mathrm{~b}$ & 1.4 & 1.4 & $\mathrm{a}$ \\
\hline $\mathrm{BF}$ & 28 & 11 & $\mathrm{ab}$ & 34 & 12 & $\mathrm{a}$ & 34 & 16 & $\mathrm{a}$ & 24 & 10 & $a b$ & 28 & 16 & $\mathrm{ab}$ & 18 & 9.7 & $\mathrm{~b}$ & 29 & 13 & $\mathrm{ab}$ \\
\hline $\mathrm{FF}$ & 5.7 & 6.3 & $\mathrm{a}$ & 19 & 8 & $\mathrm{~b}$ & 20 & 12 & $\mathrm{~b}$ & 37 & 8.3 & $\mathrm{c}$ & 17 & 11 & bd & 9.9 & 4.3 & $\mathrm{~d}$ & 38 & 11 & $\mathrm{c}$ \\
\hline $\mathrm{O}$ & 33 & 14 & $\mathrm{ac}$ & 36 & 16 & $\mathrm{a}$ & 31 & 16 & $a b c$ & 20 & 7.2 & $\mathrm{bc}$ & 44 & 18 & $\mathrm{a}$ & 38 & 18 & $\mathrm{a}$ & 21 & 9 & $\mathrm{c}$ \\
\hline PR & 19 & 12 & $\mathrm{a}$ & 2.1 & 2.9 & $\mathrm{bc}$ & 0.4 & 0.8 & $\mathrm{~b}$ & 6.2 & 6.4 & $\mathrm{c}$ & 3.4 & 3.9 & $\mathrm{c}$ & 22 & 13 & $\mathrm{a}$ & 2.2 & 1.7 & $\mathrm{c}$ \\
\hline AP & 0.3 & 0.8 & $\mathrm{ab}$ & 0.4 & 0.8 & $a b$ & 2.7 & 8 & $a b$ & 0 & 0 & $\mathrm{a}$ & 0.8 & 0.2 & $\mathrm{~b}$ & 0.4 & 0.6 & $\mathrm{~b}$ & 0.4 & 0.7 & $\mathrm{~b}$ \\
\hline $\mathrm{DL}+\mathrm{U}$ & 0.5 & 0.7 & $\mathrm{a}$ & 1.5 & 1.9 & $\mathrm{a}$ & 0.2 & 0.4 & $\mathrm{~b}$ & 0 & 0 & $\mathrm{~b}$ & 0.4 & 0.7 & $\mathrm{bc}$ & 1.9 & 2.7 & $\mathrm{ac}$ & 0.7 & 0.5 & $\mathrm{ac}$ \\
\hline \multicolumn{22}{|c|}{ Colonizer -Persister groups } \\
\hline cp 1 & 0.6 & 1.7 & $a b$ & 0.7 & 1 & $\mathrm{a}$ & 0 & 0 & $\mathrm{bc}$ & 0.3 & 0.5 & $\mathrm{ac}$ & 0 & 0 & $\mathrm{bc}$ & 1.1 & 2 & $\mathrm{a}$ & 0.9 & 1.2 & $\mathrm{a}$ \\
\hline cp 2 & 23 & 13 & $\mathrm{ad}$ & 30 & 16 & $\mathrm{a}$ & 34 & 23 & ad & 12 & 5.7 & $\mathrm{bc}$ & 29 & 18 & $\mathrm{ad}$ & 22 & 9.8 & $\mathrm{ad}$ & 21 & 13 & $\mathrm{~cd}$ \\
\hline cp 3 & 7.1 & 9.1 & $\mathrm{ac}$ & 6.3 & 5.1 & $\mathrm{ac}$ & 9.3 & 5.9 & $a b$ & 16 & 11 & $\mathrm{~b}$ & 5.4 & 3.6 & $\mathrm{ac}$ & 5.5 & 3.7 & ac & 4.6 & 4.9 & $\mathrm{c}$ \\
\hline cp 4 & 39 & 15 & $\mathrm{ac}$ & 30 & 14 & $\mathrm{ac}$ & 14 & 7 & $\mathrm{~b}$ & 28 & 7 & $\mathrm{a}$ & 30 & 15 & ae & 40 & 11 & ce & 55 & 13 & $\mathrm{~d}$ \\
\hline cp 5 & 15 & 14 & $\mathrm{ac}$ & 24 & 15 & $a b$ & 28 & 15 & $a b$ & 31 & 7.8 & $\mathrm{~b}$ & 27 & 21 & $a b$ & 20 & 14 & $a b$ & 9.3 & 4.6 & $\mathrm{c}$ \\
\hline pp 2 & 5.7 & 9 & $\mathrm{a}$ & 5.1 & 3.4 & $a b$ & 7.8 & 6.3 & $a b$ & 4.9 & 2 & $\mathrm{a}$ & 6.1 & 4 & $\mathrm{ab}$ & 8.3 & 3.3 & $\mathrm{~b}$ & 5.7 & 4.7 & $a b$ \\
\hline pp 3 & 5.4 & 9.2 & $a b$ & 1.5 & 2.1 & $\mathrm{a}$ & 4.6 & 7.6 & $a b c$ & 8.1 & 11 & $\mathrm{~b}$ & 0.7 & 1 & $\mathrm{~cd}$ & 1.1 & 2 & $\mathrm{~cd}$ & 0.4 & 1 & $\mathrm{~d}$ \\
\hline pp 4 & 2.4 & 4.4 & $a b$ & 0.4 & 0.7 & $\mathrm{a}$ & 0 & 0 & $\mathrm{a}$ & 0 & 0 & $\mathrm{a}$ & 0 & 0 & $\mathrm{a}$ & 0 & 0 & $\mathrm{a}$ & 2.4 & 2.9 & $\mathrm{~b}$ \\
\hline pp 5 & 0 & 0 & $\mathrm{a}$ & 0 & 0 & $\mathrm{a}$ & 0 & 0.1 & $\mathrm{a}$ & 0 & 0 & $\mathrm{a}$ & 0 & 0 & $\mathrm{a}$ & 0 & 0 & $\mathrm{a}$ & 0 & 0 & $\mathrm{a}$ \\
\hline MI & 3.51 & 0.35 & $\mathrm{a}$ & 3.47 & 0.45 & $\mathrm{a}$ & 3.43 & 0.6 & $\mathrm{a}$ & 3.89 & 0.18 & $\mathrm{~b}$ & 3.60 & 0.52 & $\mathrm{ab}$ & 3.56 & 0.42 & $a b$ & 3.55 & 0.33 & $\mathrm{a}$ \\
\hline$\Sigma \mathrm{MI}$ & 3.38 & 0.37 & $\mathrm{a}$ & 3.39 & 0.44 & $\mathrm{a}$ & 3.30 & 0.56 & $\mathrm{a}$ & 3.72 & 0.15 & $\mathrm{~b}$ & 3.50 & 0.51 & $\mathrm{ab}$ & 3.44 & 0.43 & $\mathrm{ab}$ & 3.45 & 0.3 & $\mathrm{a}$ \\
\hline MI 2-5 & 3.55 & 0.35 & $\mathrm{a}$ & 3.53 & 0.46 & $\mathrm{a}$ & 3.44 & 0.6 & $\mathrm{a}$ & 3.9 & 0.18 & $\mathrm{~b}$ & 3.61 & 0.52 & $\mathrm{ab}$ & 3.64 & 0.36 & $\mathrm{ab}$ & 3.60 & 0.34 & $\mathrm{a}$ \\
\hline PPI & 2.51 & 0.38 & $\mathrm{abc}$ & 2.30 & 0.25 & $\mathrm{~b}$ & 2.33 & 0.34 & $\operatorname{cbd}$ & 2.56 & 0.21 & $\mathrm{cb}$ & 2.09 & 0.11 & $\mathrm{~d}$ & 2.08 & 0.12 & $\mathrm{~d}$ & 2.43 & 0.42 & $\mathrm{~b}$ \\
\hline CI & 79.8 & 38.1 & $a b c$ & 54.3 & 33.2 & $\mathrm{ac}$ & 94.3 & 18.1 & $\mathrm{~b}$ & 85.8 & 25.6 & bd & 88.3 & 20.1 & bd & 76.1 & 33.1 & $\mathrm{ad}$ & 41.3 & 19.9 & $\mathrm{c}$ \\
\hline EI & 12.9 & 18.4 & $\mathrm{a}$ & 33.6 & 13.8 & $\mathrm{~b}$ & 22.6 & 7.65 & $\mathrm{~cd}$ & 31.8 & 11 & $\mathrm{~b}$ & 19.3 & 14.1 & $\mathrm{ac}$ & 30.8 & 13.6 & bd & 36.5 & 18.6 & bd \\
\hline SI & 91.3 & 5.84 & $\mathrm{a}$ & 87.6 & 12.1 & $\mathrm{a}$ & 82.3 & 20.5 & $\mathrm{a}$ & 95.7 & 1.63 & $\mathrm{~b}$ & 89.8 & 8.07 & $\mathrm{ab}$ & 92.2 & 4.71 & $\mathrm{ac}$ & 92.5 & 6.89 & $\mathrm{bc}$ \\
\hline
\end{tabular}

Feeding types: Plant-feeding (PF, a-f subgroups according to Yeates et al., 1993), algal-feeding (AF), bacterial-feeding (BF), fungal-feeding (FF), omnivores (O), predators (PR), animal parasites (AP), Dauer larvae and unidentified (DL+U); in a given line numbers followed by different letters differ significantly $(\mathrm{p}<0,05, \mathrm{M}-\mathrm{W}$ test $)$. The mean relative abundance for the trophic and c-p groups was expressed as $\%$ from $100 \mathrm{~cm}^{3}$ soil. 
along the first ordination axes by the PCA method. Nematode assemblages from the dvM site were most similar with those from $\mathrm{pM}$ and $\mathrm{t} 2 \mathrm{M}$ sites (65 and $63 \%$, respectively). On the contrary, the greatest dissimilarity was exhibited between the nematode communities of t1M plot compared with t3M (23\%) and uM (27\%) sites.

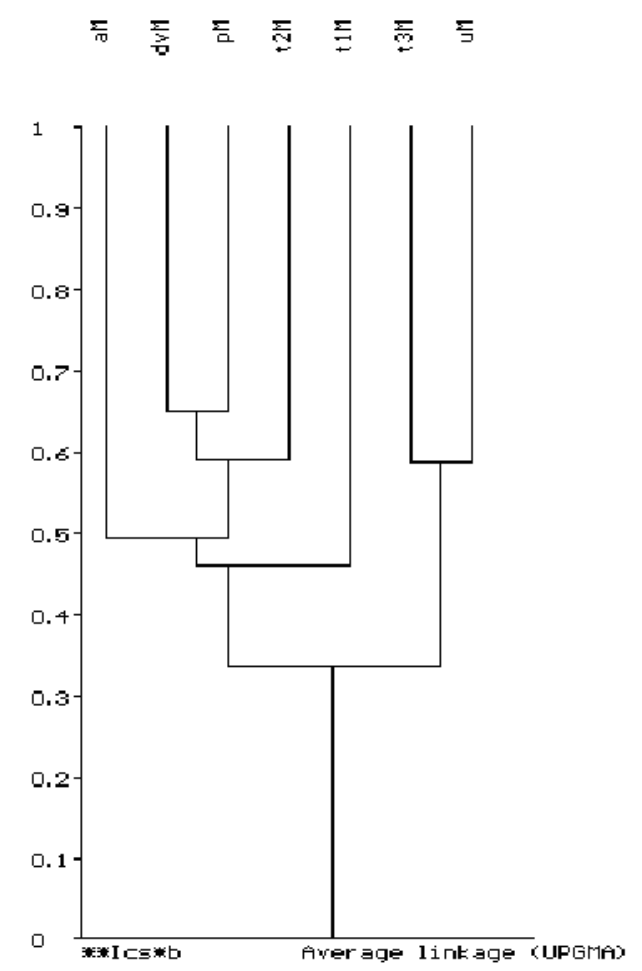

Fig. 2. Dendrogram (UPGMA) representing the classification of the studied $P$. mugo sites.

\section{Functional groups and community indices}

The relationships among the different ecological parameters (functional groups, indices, etc.) and the nematode assemblages' distribution along the first and second axes of the ordination biplot are presented on the Fig. 3. The communities of $\mathrm{uM}, \mathrm{t} 2 \mathrm{M}, \mathrm{t} 3 \mathrm{M}$ sites can be characterised with lowest nematode abundance, generic richness and Shannon diversity index (see Table 3 for details). Two of them ( $2 \mathrm{M}$ and $\mathrm{t} 3 \mathrm{M})$ were situated next to the ski runs, while $\mathrm{uM}$ was close to a touristic tour pathways located in a stony area on a steep slope. Among the communities having significantly higher abundance, richness and diversity, two were next to the ski runs (pM, t1M), while the other two were situated in areas being under low anthropogenic pressure ( $\mathrm{dvM}$ and $\mathrm{aM})$. In comparison, the nematode communities from Vitosha, also located in vicinity of ski runs, exhibited the lowest values for the relative abundance and diversity - av. 327 specimens $/ 200 \mathrm{~cm}^{3}$ soil, av.1.91 for H' and av. 15 genera per sample (Lazarova, 2000).

Trophic group distribution varied significantly among the studied sites. In general, the communities of all plots had a high proportion of omnivorous (aver. values $20-44 \%$ ) and bacterial feeding $(18-34 \%)$ nematodes. Furthermore, plant feeders (groups belonging to $1 \mathrm{~b}$-e) were with relatively low abundance in all sites (aver. values $3.1-10 \%$, lower values assigned to $\mathrm{t} 2 \mathrm{M}$ and $\mathrm{t} 3 \mathrm{M}$ sites). Some assemblages were dominated by fungal feeders (37 and 38\% for $\mathrm{t} 1 \mathrm{M}$ and aM, respectively) but in others the proportion of predators was significantly higher (19 and $22 \%$ for uM and $\mathrm{t} 3 \mathrm{M})$. These sites represented communities with the lowest relative abundance and generic richness, coupled with the highest proportion of omnivorous nematodes (33, 38 and $44 \%$ for $u M, t 2 M$ and $\mathrm{t} 3 \mathrm{M}$ ).

Similarly, omnivorous and bacterial feeding nematodes dominated the nematode communities from Vitosha Mt., although their trophic structure showed a characteristic feature - predators and plant feeders were present in similar proportions (ca. $20 \%$ ), while fungal feeders had very low number (Lazarova, 2000). In contrast to these observations, in the P. mugo site from Rila Mt. the plant and bacterial feeding nematodes prevailed (30 and $27 \%$ ), and the other trophic groups were presented in comparable shares (16, 14 and $12 \%$ for predatory, fungal feeders and omnivorous nematodes) (Ilieva, 2007). These differences could be addressed to the seasonal variations - the samples from Rila site have been collected in autumn.

The bacterial feeders and omnivorous nematodes were represented by the highest number of genera, the generic richness of the first group varying in wide ranges (Fig. 4).

The distribution of colonizer-persistor groups exhibited higher resemblance among sites for both free-living and plant-feeding nematode taxa (Table 3). The proportion of enrichment opportunists (cp1) was very low for all sites, while the general opportunists (cp2) were well presented

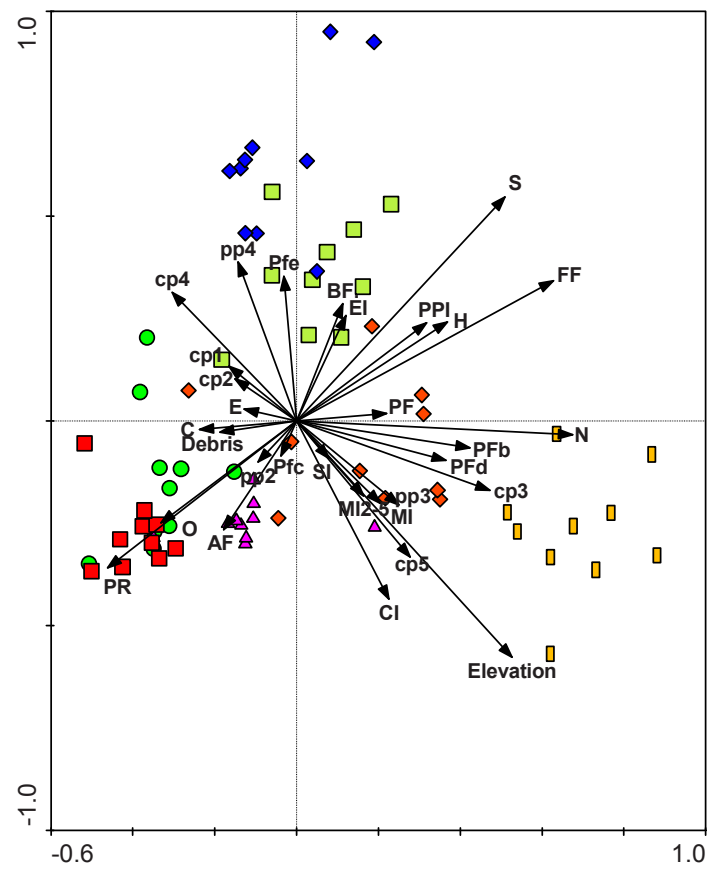

Fig. 3. Principal Component Analysis of the relationship between the ecological parameters (functional groups and indices) and the nematode assemblages' distribution (abbreviations are the same as in Table 3.). 
$(12-34 \%)$ and even dominated in some sites (dvM and $\mathrm{pM}$ ). The overall abundance of persistors (cp4 and cp5) was generally high $(42-64 \%)$. The cp4 group of nematodes dominated at several sites ( $\mathrm{uM}, \mathrm{t} 2 \mathrm{M}, \mathrm{t} 3 \mathrm{M})$ and prevailed significantly at aM (55\%), while nematodes of cp5 reached the highest relative abundance $(31 \%)$ at t1M. The generic richness of two life strategy groups (cp4 and pp2) had higher average values for all plots (Fig. 5). blages from Rila, which had the highest reported value (2.71).

Further, the variations of the average values for enrichment and structure indices fall in very narrow limits (EI, $12.9-$ 36.5 and SI, 82.3 - 95.7). Data from each replicate plotted along the enrichment and structure trajectories related most of P. mugo sites to C quadrant (Ferris et al., 2001). Thus, using functional guilds, the soil food webs in the studied

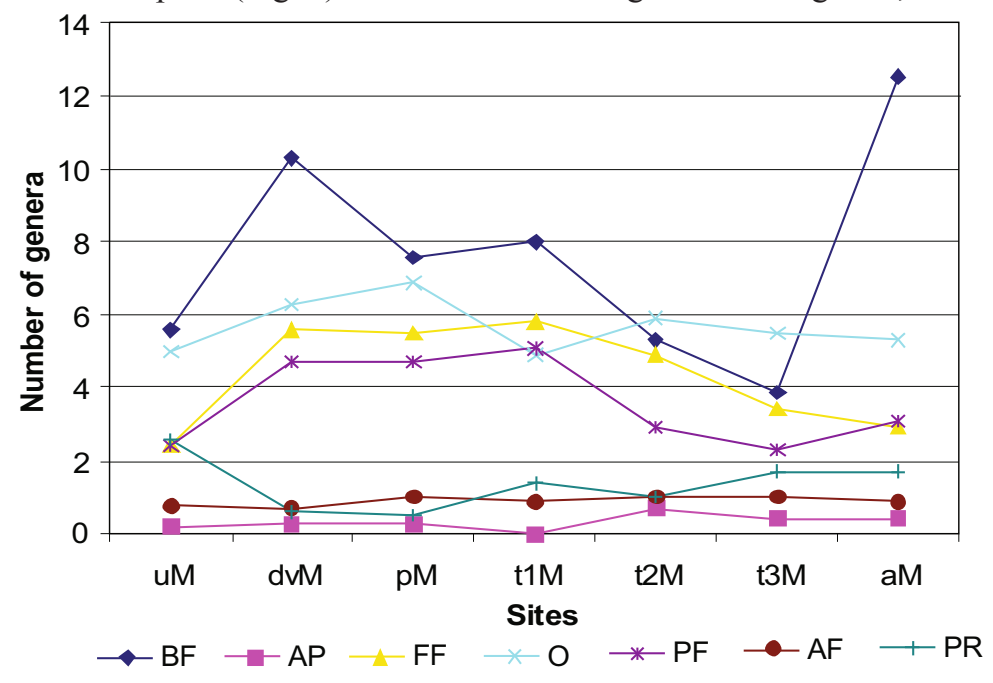

Fig. 4. Generic richness of nematode trophic groups inhabiting Mountain Pine.

The indices characterising the maturity of nematode communities (MI, इMI, MI2-5) showed similar trends of variation among sites with significantly higher values detected for $\mathrm{t} 1 \mathrm{M}$ site $(\mathrm{p}<0.05$, Table 3$)$. Sites having communities with the lowest nematode abundance and diversity ( $2 \mathrm{M}, \mathrm{t} 3 \mathrm{M}$ ) revealed the lowest PPI values compared to the other sites. The values of the maturity indices recorded in previous investigations (Lazarova, 2000; Ilieva, 2007) varied within the ranges revealed for the majority of plots at Pirin Mt, excluding PPI for assem- plots can be characterised as structured, environment as undisturbed, with low to moderate enrichment (resourcelimited) and stable. Similar soil food web conditions were identified in P. mugo site at Vitosha Mt (Lazarova, unpublished), while on Rila Mt the food web has been characterised as maturing (Ilieva, 2007). On the opposite to SI and EI, Channel index (CI) varied considerably among the studied sites (Table 3) with lowest and highest values belonging to aM and pM sites, respectively (av. 41.3 vs 94.3). Consequently, the decomposition soil food webs in most of

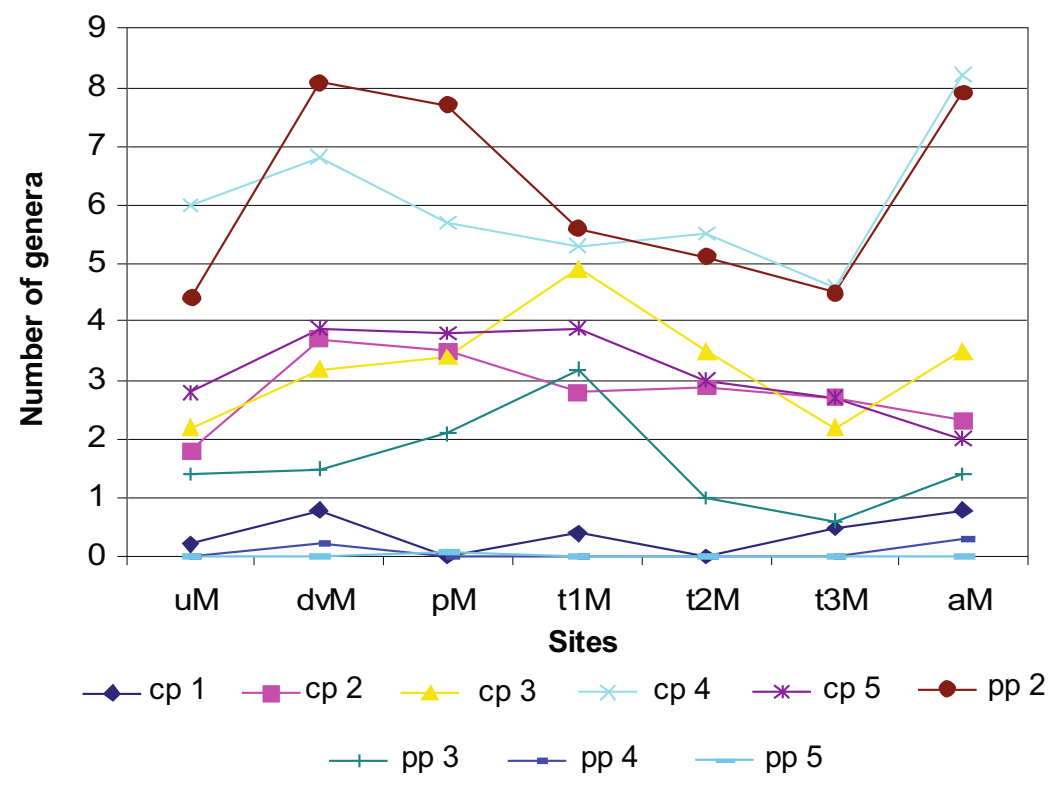

Fig. 5. Generic richness of nematode functional groups inhabiting Mountain Pine. 
the sites were dominated by fungal feeders. On the basis of CI, only two plots (dvM and $\mathrm{aM}$ ) exhibited similarity with P. mugo site from Rila Mt (Ilieva, 2007), which reflected the nature of the decomposition pathway dominated by bacterial feeding nematodes.

\section{Concluding remarks}

Nematode communities of $P$. mugo habitat from northern Pirin Mountain are very diverse. Although the trophic structure differed among sites, it was dominated by omnivorous and bacterial feeders coupled with a low proportion of plant feeding nematodes. The high values of Maturity indices, and significant participation (key position) of persistors indicated a relative stability of the environment and undisturbed conditions in all sites. This was supported by the nematode faunal analysis characterising the decomposition channels as fungal dominated and food web as structured, the habitat -undisturbed with limited resources. Further, three types of marginal sites can be differentiated: the first represented assemblages with the lowest relative abundance and generic richness, dominated by omnivorous and predatory nematodes; the second one - with the highest abundance, dominated by fungal feeders and the third one with the highest generic richness and strong prevalence of persistors. The rest of the sites occupied an intermediate position regarding community parameters and indices. No clear correlation between the nematode assemblages' characteristics and the proximity of the site to the ski runs was observed.

\section{Acknowledgements}

This research was partly supported by the grants $1405 / 04$ and INI 03/01/08.2005 (BioCORE) from The National Fund "Scientific Investigations".

The authors gratefully acknowledge Dr. T. Tonchev, University of Forestry, Sofia for identifying the plant cover and botanist E. Kachaunova, Insitute of Botany, Bulgarian Academy of Sciences for the identification of the herbarium. We are thankful to Dr L. Penev, CLGE, for the critical reading of the manuscript and helpful suggestions.

\section{References}

BAev, P., Penev, L. (1995): BIODIV: Program for calculating biological diversity parameters, similarity, niche overlap and cluster analysis. Exeter Software, New York BONGERS, T. (1990): The maturity index: an ecological measure of environmental disturbance based on nematode species composition. Oecologia, 83: $14-19$

De Goede, R. G. M., Bongers, T. (1994): Nematode community structure in relation to soil and vegetation characteristics. Appl. Soil Ecol., 1: 29 - 44

EngelmanN, H. D. (1978): Dominanzklassifizierung von Bodenarthropoden. Pedobiologia, 18, (5/6): 378 - 380

Ferris, H., Bongers, T., DE Goede, R. G. M. (2001): A framework for soil food web diagnostics extension of the nematode faunal analysis concept. Appl. Soil Ecol., 18: $13-29$
Hoschitz, M. (2003): Moss-living nematodes from an Alpine summit (Dachstein, Austria). Vehr. Zool.-Bot. Ges. Österreich, 140: 93 - 98

Hoschitz, M., Kaufmann, R. (2004): Soil nematode communities of Alpine summits - site differentiation and microclimatic influences. Pedobiologia, 48: 313 - 320 Hoschitz, M., KaUfMANN, R. (2004): Nematode community composition in five alpine habitats. Nematology 6(5): $737-747$

IlieVA, ZH. (2007): Soil nematodes communities from the Biosphere Reserve "Parangalica", PhD thesis. Sofia

JøRGENSEN, H. (2006): NOBANIS - Invasive Alien Species Fact Sheet-Pinus mugo.- From: Online Database of the North European and Baltic Network on Invasive Alien Species-NOBANIS www.nobanis.org

Korthals, G. W., De Goede, R. G. M., Kammenga, J. E., BONGERS, T. (1996): The maturity index as an instrument for risk assessment of soil pollution. In: Straalen, N.M. and van Krivolutsky, D.A. (Eds.), Bioindicator Systems for Soil Pollution, Kluwer, Dordrecht

Korthals, G. W., Alexiev, A. D., Lexmond, T. M., KAMMEnGA, J. E., BONGERS, T. (1996a): Long-term effects of copper and $\mathrm{pH}$ on the nematode community in an agroeco-system. Environ. Toxicol. Chem., 15: 979 - 985

Korthals, G. W., VAn Der Ende, A., VAn MEgen, H., LeXMOnd, T. M., KAMmenga, J. E., Bongers, T. (1996b): Short-term effects of cadmium, copper, nickel and zink on soil nematodes from different feeding and life-strategies groups. Appl. Soil Ecol., 4: 107 - 117

KREBS, C.J. (1989): Ecological methodology. Harper Collins Publishers, New York

Kuzniar, A., Gheorghe, I., Krecmer, V., Niemtur, S., PeneV, L., Peneva, V., Richards, C., Scharffova, K. \& TWARDY, S. 2004. 7. Uplands: 75-87. In: YounG, J., Halada, L., Kull, T., KuZniar, A., Tartes, U. \& Watt, A. (Eds) Conflicts between human activities and conservation of biodiversity in agricultural landscapes, grasslands, forests, wetlands and uplands in the Acceding and Candidate Contries (ACC), CEH, Glassel, Scotland, 97 pp. BIOFORUM report.

LAZAROVA, S. (2000): Comparative analysis on the biodiversity of phytonematode assemblages from forest ecosystems. PhD thesis

NeHER, D. A. (2001): Role of nematodes in soil health and their use as indicators. J. Nematol., 33: $161-168$

NiBlACK, T. L. (1989): Application of Nematode Community Structure Research to Agricultural Production and Habitat Disturbance. J. Nematol., 21: 437 - 443

RAEV, V. (ED). (2005). Inventory and strategy for sustainable management and protection of of virgin forests in Bulgaria. FRI-BAS-KNNV

Ruess, L., Michelsen, A., Jonasson, S. (1999a): Simulated climate change in subarctic soils: responses in nematode species composition and dominance structure. Nematology, 1: $513-526$

Ruess, L., Michelsen, A., Schmidt, I. K., Jonasson, S. (1999b): Simulated climate change affecting microorganisms, nematode density and biodiversity in subarctic soils. 
Plant soil, 212: $63-73$

SEINHORST, J. W. (1959): A rapid method for transfer of nematodes from fixative to anhydrous glycerine. Nematologica, 4: $67-69$

SOHLENIUS, B., BoströM, S. (1999a): Effects of global warming on nematode diversity in a Swedish tundra soil - a soil transplantation experiment. Nematology, 1: 695 - 709

SOHLENIUS, B., BoströM, S. (1999b): Effects of climate change on soil factors and metazoan microfauna (nematodes, tardigrades and rotifers) in a Swedish tundra soil - a soil transplantation experiment. Appl. Soil Ecol., 12: 113 - 128

STATSOFT, INC. (2007): STATISTICA (data analysis software system), version 8.0

TER BRAAK, C. J. F. (1996): Unimodal models to relate species to environment. DLO-Agricultural Mathematics Group, Wageningen

RECEIVED APRIL 16, 2008
TER BraAk, C. J. F., SMilauer, P. (2002): CANOCO Reference manual and CanoDraw for Windows User's guide: Software for Canonical Community Ordination (version 4.5). Microcomputer Power (Ithaca, NY, USA)

TSARYK, I., KOZLOWSKI, M. (1999): Phytoparasitic nematodes of Pinus mugo Turra in the Highlands of the Ukrainian Carpathians. Wiad. Parazytol., 45(4): 543 - 544

Yeates, G. W., Bongers, T., DE Goede, R. G., Freckman, D. W., Georgieva, S. S. (1993): Feeding habits in soil nematode families and genera - an outline for soil ecologists. J. Nematol., 25: $315-331$

YeAtes, G. W. (1994): Modification and qualification of the nematode Maturity index. Pedobiologia, 38: 97 - 101 YEATES, G. W. (2003): Nematodes as soil indicators: functional and biodiversity aspects. Biol. Fert. Soils, 37: $199-$ 210

ACCEPTED JANUARY 14, 2008 